ECONOMICS

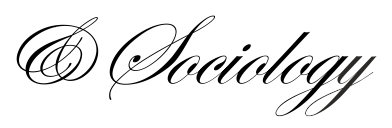

\author{
Rafał Kasperowicz, \\ Poznan University of Economics \\ and Business, \\ Poznań, Poland, \\ E-mail: \\ rafal.kasperowicz@ue.pornan.pl
}

Marcin Pinczyński,

Universidad Pontificia Comillas ICAI,

Madrid, Spain,

E-mail:

pincsynskimarcin@gmail.com

Aviral Tiwari,

Montpellier Business School,

Montpellier, France,

E-mail:aviral.eco@gmail.com

Lukasz Nawrot,

Poznan University of Economics

and Business,

Poznañ, Poland,

E-mail:l.nawro@@u.poznan.pl

Received: March, 2017

1st Revision: July, 2017

Accepted: October, 2017

DOI: $10.14254 / 2071-$

789X.2017/10-4/14

JEL Classification: Q4, E03, E37, O10, N70
Kasperowicz, R., Pinczyński, M., Tiwari, A., Nawrot, Ł. (2017). Reengineering of Electricity Market Monitoring. Economics and Sociology, 10(4), 175-188. doi:10.14254/2071-789X.20171/10-4/14

\section{REENGINEERING OF ELECTRICITY MARKET MONITORING}

\begin{abstract}
The main goal of this paper is to present new indices and a new structure of the electricity market monitoring system. The proposed structure, along with the analytical methods and indices applied to this structure, could be used from the viewpoint of both market operator and market participants. This paper also describes, in a rather simplified manner, the algorithms of market monitoring on the case study of Spanish electricity market.
\end{abstract}


Štreimikienè, 2016; Brożyna et al., 2016; Strielkowski et al., 2017). Supervisory actions are important for the system operator as well as regulatory commissions in their ensuring efficient market performance. This article omits the basic concepts and issues of electricity markets functioning and monitoring, since a detailed literature review as well as theoretical introduction to these issues can be found in our previous article "Overview of electricity market monitoring" (Pinczynski \& Kasperowicz, 2016) and the interested reader is advised to read these two papers as a whole entity. After a broad study of the literature related to monitoring and analysis of the electricity market we can conclude that there is lack of information concerning the techniques to be used for monitoring economic parameters at the markets with a structure similar to Spanish market. So, there is a need to propose new indices and/or modifications to the already existing ones at Spanish electricity market, same applies to the markets similar to Spanish one.

This paper firstly introduces a new index for monitoring of economic parameters. In section II application of this index and methods for monitoring Spanish electricity market, considering publicly available data sources, are proposed. In the last part, a new monitoring system structure along with new algorithms, designed specifically for Spanish electricity market, are presented.

\section{New index}

As it was discussed in the previous paper (Pinczynski \& Kasperowicz, 2016), the existing methods have a limited applicability to the real power systems, due to specific data requirements, or the theoretical limitations. The proposed indexes can be readily applied to the existing system (Spanish power system) and provide relevant information about the market behavioral.

Bid price of cleared energy monitoring index (BPceM) depicts the relation of the difference between the weighted average price of cleared energy for a certain technology during the period of one day and the weighted average price of cleared energy for all the technologies during the period of one day, to the weighted average price of cleared energy for all the technologies during the period of one day.

$$
\text { BPceM }=\frac{w P c E i, t-w P c E j, t}{w P c E j, t}
$$

$w P c E i, t$ - weighted average price of cleared energy for a certain technology in time $\mathrm{t}$, $w P c E j, t$ - weighted average price of cleared energy for all the technologies in time $\mathrm{t}$.

This index determines, whether a certain technology shall be classified as inflating or lowering the weighted average price. If the index value is negative, it implies that the weighted average price of the offers for this particular technology during a certain day was lower than the weighted average price for all the technologies. On a contrary, if the index value is positive, it evidences that this particular technology might have triggered an increase of the energy final price. Finally, if the values amount to around zero, it indicates that the weighted average price of cleared energy was approximate to the weighted average price for the whole system. The values are expressed as a percentage and they reflect the number of percentage points by which the cleared energy price (relating certain technology) rises or falls with regard to the cleared energy price for all the technologies.

The abovementioned index could be applied also in order to determine the agent or the group of agents that had impact on the energy price change, as regards certain technology or taking into account the whole system. If the weighted average price of cleared energy for a certain technology is replaced by the weighted average price for a certain agent on a certain 
day, and if the weighted average price of cleared energy for the whole system is replaced by the weighted average price of cleared energy for the whole technology, then the presented index could be helpful in seeking the agents which have triggered an increase or decrease of the energy final price as regards a certain technology. In such case the index would present an increase or decrease of the weighted average price with regard to the weighted average price of cleared energy for the whole system, and would be expressed as a percentage. The interpretation of the obtained measurement results shall be analogous to the interpretation of the index focused on technology.

Furthermore, the abovementioned index could be also modified in order to monitor relations between the weighted average prices for different agents or groups of agents. Such modified index could be helpful in monitoring the relations between those agents or groups of agents that have the greater influence on the final price level.

One of the primary advantages of this index is the fact that it uses the weighted average prices of energy with regard to the amount of energy. The monitoring of prices, without considering the quantitative aspect, is in the most cases insufficient. In such case, if there is only one highly priced offer regarding a small amount of energy, it could significantly distort the results. Nevertheless, this problem has been resolved thanks to the application of the weighted average price. The presented index aim at presenting the influence of a certain technology, agent or group of agents on the final energy price.

The index introduces three primary pieces of information:

if the index value is positive, then the examined group might have triggered an increase of the final price,

if the index value is negative, then the group might have contributed to the decrease of the final price, whereas,

if the value amounts to about zero, it implies that the weighted average price of the offered energy was approximately to the weighted average price of the energy with regard to the whole analyzed system.

The index value is expressed as a percentage and it depicts the differences between the weighted average prices of cleared energy with regard to the examined different groups. This index could be applied over a horizon of one hour, one day or one week, and even over some longer horizons, if there is such a need.

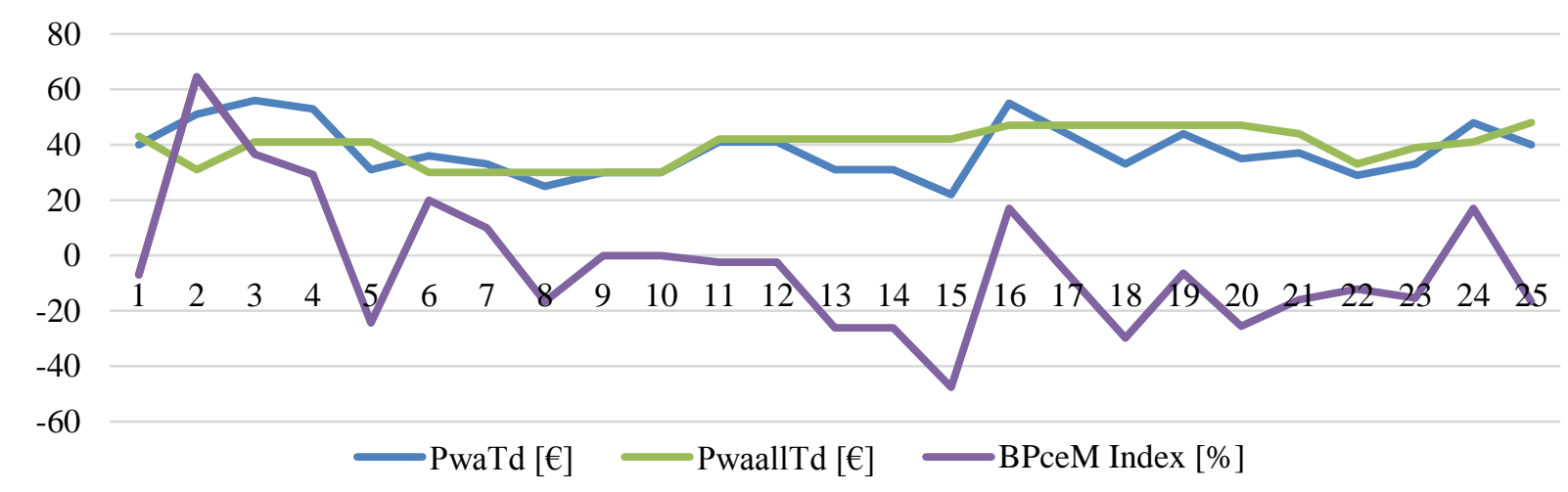

Figure 1. The graph presents the graphical representations of the bid price of cleared energy monitoring index

Source: own calculation. 
The Figure 1 refers to period of 25 days, whereas the weighted average price of cleared energy for a certain agent is marked in blue, the weighted average price of cleared energy with regard to the whole system - in green, whereas the indications of the bid price of cleared energy monitoring index, expressed as a percentage, are marked in violet. BPceM index shows when and by how many percent the examined technology differed from the final weighted average price for the whole system. Furthermore, it indicates also in what manner the agent could have influenced the final energy price (increase, decrease, maintaining the average level).

It is evidenced in the graph that on the first day the weighted average price as regards the examined agent was by a few percent lower than the price regarding the whole system. Further, on the next day the weighted average price increased by almost $55 \%$ and such high price level tended to maintain until the fifth day, on which the weighted average price decreased by $10 \%$ of the weighted average price with respect to the whole system.

Moreover, from ninth to twelfth day the index value amounted to approximately 0 , which implies that the offers accepted in the market, made by a certain agent, were broadly in the same range as the cleared energy price for the whole system. The abovementioned pieces of information are helpful in identifying the periods during which the particular agent could have had some impact on: inflating the final price (days 2-4, 6-7, 16, 24), lowering the final price (days 5, 13-15, 18-23), maintaining the average level of prices for the whole system (days 9-12).

\section{Indexes and methods of the monitoring of Spanish electricity market}

\subsection{Indexes implemented directly}

The public availability of the source data makes possible the direct (without modifications) application of the following indexes:

- Hertlndahl- Hirschman Index,

- Residual Supply Index,

- Price-Cost Margin Index,

- Markup Ability,

- Market clearing price distribution index,

- Earnings in Total Income,

- Earnings in Price Markup (Pinczynski \& Kasperowicz, 2016).

\subsection{The analytical methods that could be applied to the monitoring of Spanish market}

\subsubsection{Screening thresholds}

Through the application of appropriate algorithms to analyzing Spanish source data, it is possible to obtain information regarding the characteristics of bids (offers) submission by particular agents (the examined groups could be classified by technologies or groups of agents). If the data is grouped properly, relevant filters could be applied with the aim of monitoring the behavior of a particular agent, group of agents or a particular technology (bidding patterns monitoring). The abovementioned method could be realized mainly with regard to Behavioral monitoring, however, it could be also helpful in detecting the first signals of creating the market power. As a rule, the monitoring of market efficiency requires some wider analyzes than screening thresholds (Patton, 2003; Gan \& Bourcier, 2002; Galarza et al., 2006). 
The monitoring of the price ranges of the weighted average energy prices with regard to the amount of energy of particular agents, groups of agents or technologies could easily indicate which agents or technologies are responsible for a change of the final energy price (see Appendix A). As a result of selecting the particular agent, group of agents or technology, it is possible to conduct a detailed analysis of submitted bids by virtue of studying the graphs presenting directly all the bids related to the examined group (see Figure 2).

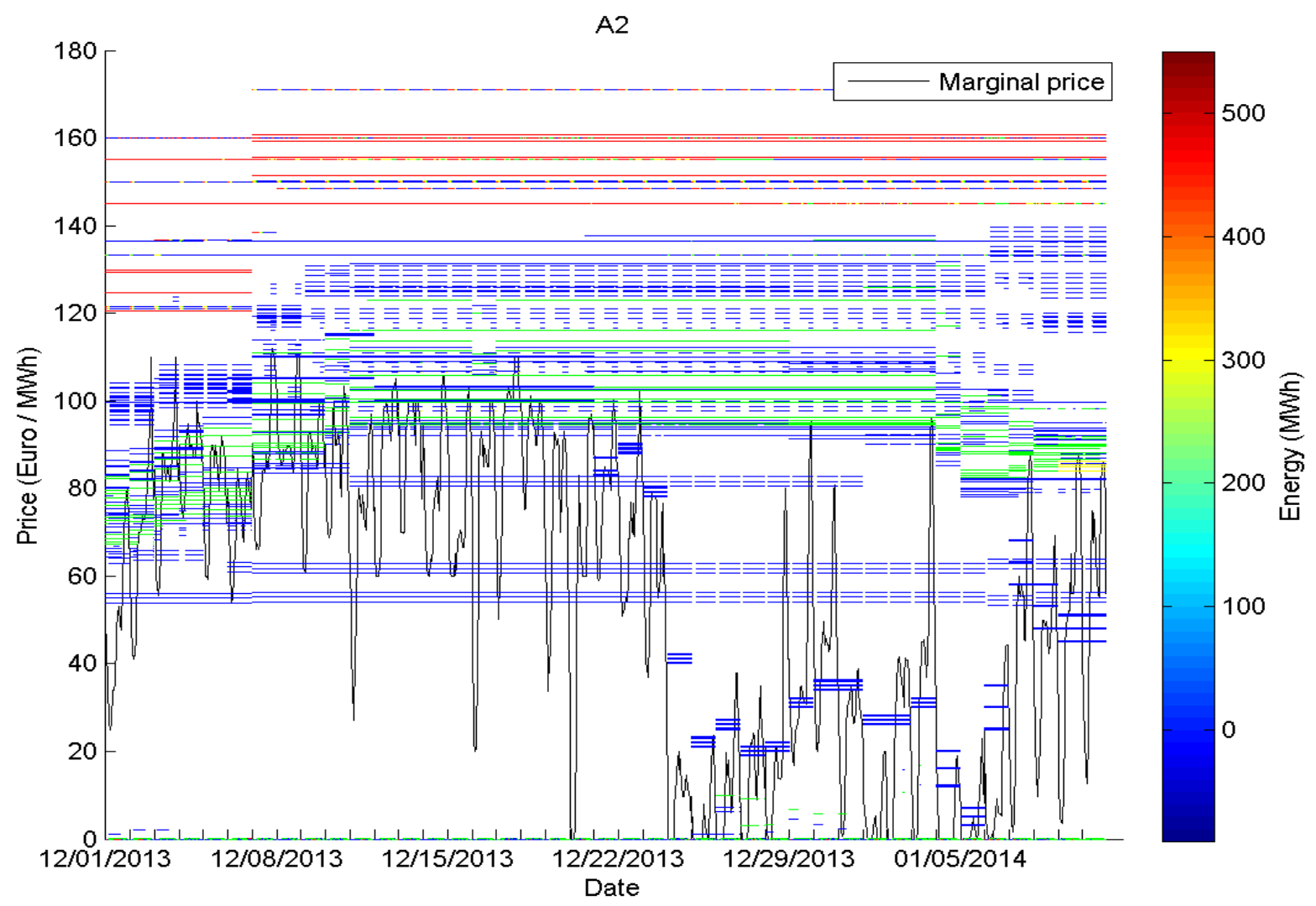

Figure 2. The graphical representation of the submitted bids of agent A2, period from $12 / 01 / 2013$ to $01 / 05 / 2014$

Source: Own calculation.

Figure 2 is one of the core graphs that presents the behavior of a particular agent and, as a consequence, is one of the elements of participants behavioral monitoring. Furthermore, the application of relevant filters, that monitor the parameters of bids, would make it possible to monitor the situations that are conducive to creating market power, to register current trends in the market, to detect abnormal behavioral of market participants (in order to detect them it is crucial to define the parameters that shall be identified as an anomaly for the system).

\subsubsection{Benchmarking}

Due to the fact that the source data regarding Spanish market is publicly available, Benchmarking could be also applied to the monitoring of market power, performers and participants behavioral monitoring. The presented method shall be qualified as a comparative analysis. The key (and at the same time quite complex) issue regarding comparative analyses is the application of the relevant group of referential models on the basis of the parameters 
describing the particular market. In order to warrant that the benchmarking results are correct, the set of reference models shall capture the characteristics and parameters of the market (such as estimate cost of fuel, outage rates, opportunity cost, etc.) in a correct and appropriate way. The application of an inappropriate reference model (e.g. inaccurate estimation of the fuel costs or of the efficiency of a particular technology) could substantially reduce the correctness of the analysis (Patton, 2003; Sole \& Bist, 1995; ETSO Report n.d.; Lisin and Strielkowski, 2014).

\section{The proposed structure of the monitoring system}

In this Section the simplified structure of the monitoring system is outlined. The structure is based on a set of control variables and analytical procedures for Spanish market monitoring. Due to the available data and the knowledge of the structure of electricity market in Spain, it was possible to present the following simplified structure of the day-ahead market monitoring system.

The system is comprised of four basic modules:

a) Data Acquisition System - is responsible for the automatic gathering and recording of the publicly available data, including information regarding the electricity market. The abovementioned module forwards the collected data to the Data Processing module;

b) Data Processing - this module aims at simple calculation of indexes and preparation of the detailed data required for further analyses within the Analysis module. Additionally, the standardization of data - i.e. the processing of data in order to have it provided in the same format and subject to the same standard of calculation (the source data is to be found in different data bases, therefore, its structure varies) - is also conducted within the Data Processing module;

c) Analysis, the purpose of the module is to conduct detailed calculation and analysis of the data. All the modules function on the basis of 3 main monitoring techniques, i.e. benchmark analysis, screening thresholds and monitoring by virtue of indexes. Each of the monitoring techniques represents a relevant sub-module, which is responsible for the realization of the calculation and data analysis in accordance with the relevant monitoring technology. The mutual relations between the modules could be observed, however, they are not indicated in the Figure 3, due to the simplified form of the scheme. Nevertheless, the full specification of monitoring techniques is presented in "Overview of electricity market monitoring" (Pinczynski \& Kasperowicz, 2016). The aim of the last sub-module (Final interpretation of the analysis) is the final integration and analysis of the results originating from submodules: detailed analysis of indexes, benchmark analysis and screening thresholds. All of those three monitoring techniques may contain information corresponding to do Market Power, market performance and behavioral monitoring. In the presented module, i.e. the pieces of information are classified pursuant to the criteria of the parameters that are being monitored. In the Final interpretation of the analysis module, the same parameters measured by different techniques are compared. Due to this fact, i.e. the fact that the same parameters are measured by different techniques, the accuracy and correctness of the conducted analyses could be boosted, as well as some internal functions could be implemented, such as the ones related to the self-diagnosis of measurement errors and errors in the analysis;

d) Reports, the results of all the analyses shall be presented in the last module of the monitoring system. Depending on particular needs, the report may be prepared in a more simplified or in a more detailed form. A simplified report includes: basic graphs regarding market parameters, warnings with regard to any possible occurrence of anomalies and alerts, which indicate the occurrence of negative situations. On the contrary, a detailed report is composed of all the information outlined in the simplified report, as well as of additional detailed 
information presenting market parameters. Both types of reports may be presented in various manners, subject to any particular needs (an email, on a webpage, as a single file etc.).

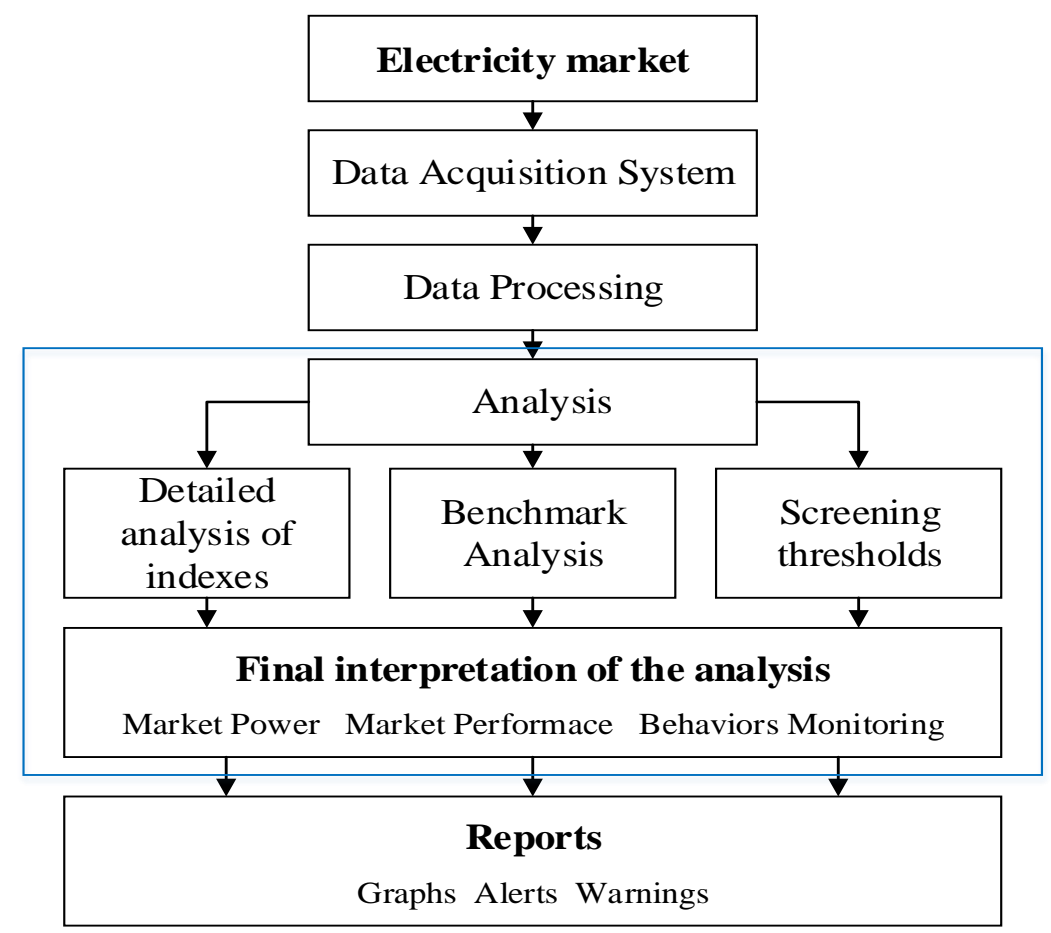

Figure 3. The structure of the day-ahead market monitoring system.

\subsection{Algorithms}

The monitoring system structure presented in Figure 3 can be supported entirely by two new algorithms that are presented in this subsection. The first algorithm uses a technique of market simulation and statistical indexes computation, whereas for the second algorithm a technique of screening thresholds shall be applied:

a) Market simulation and statistical indexes algorithm

The main aims of this algorithm is to determine whether the market functions at a competitive level and whether there are any anomalies that distort competitive market. Another important goal of this algorithm is to determine how the existing anomalies affect the market competitiveness. This algorithm collects data from the Data Processing module which is described in section 3. Afterwards, energy market simulations are simultaneously performed; further, statistical indexes are calculated. After conducting the above calculations, the reference models, necessary for the detecting anomalies and prohibited behaviours, will be determined. If the algorithm does not detect any anomalies or prohibited behaviours, a relevant report will be generated. On the contrary, when any anomaly is detected, the algorithm continues conducting further analysis, within the analysis module, which aims at examining the case in detail. Subsequent data obtained from the analysis module is transmitted to the competitive market module, which main goal is to determine whether the market is functioning on a competitive basis. If competitive market module's analysis concludes that the market is competitive, the algorithm stops and generates a relevant report. When the competitiveness of the market is not stable, the algorithm conducts further analysis to determine the impact of the detected anomaly on the competitiveness of the market - this process takes place in the module 
Identification of Impact. The final element of this algorithm is generating a report which is comprised of all the alerts warnings and graphs representing the market state.

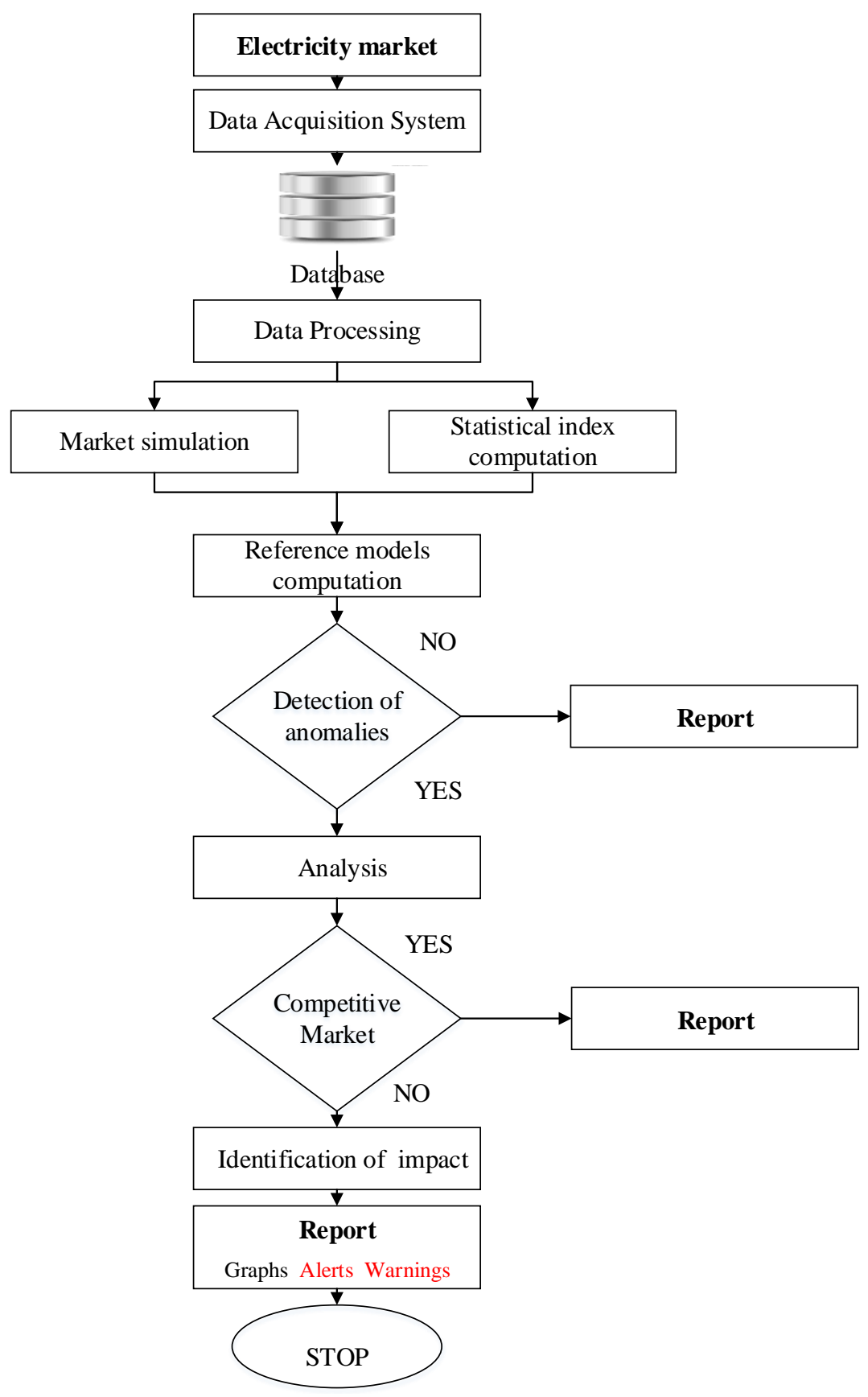

Figure 4. Market simulation and statistical indexes algorithm

b) Screening thresholds algorithm

One of the core tasks of this algorithm is to identify bids, agents or groups of agents that are responsible for an increase of the final price of electric energy (clearing price). This algorithm is based on the screening thresholds technique. The first three modules of this algorithm are the same as in the algorithm presented in Figure 4. The main features of this algorithm are three sections: 
- Screening price. This algorithm section comprises of the description of the BPcM index, presented in paragraph IV.A, which aims at determining the impact of a particular agent, technology or groups of agents on the final price of energy within a specified period of time. If the index indicates the possibility of price change or neutral impact of selected bids, the algorithm will generate a relevant report. If it detects a possible overestimation of the final energy price (clearing price), then in this section it will be analysed whether the final price could be denoted as competitive. If the tested group of bids could be allocated within a competitive price range, the algorithm will generate a report and will stop. However, if some of the prices are allocated outside the competitive range, the selected bids will be sent to the next section (Screening energy) of the algorithm.

- Screening energy. In this section, the amount of clearing energy in a competitive price range (from 0 to competitive price $\mathrm{PcC}$ ) in the selected time period is compared with the weighted average amount of energy in the previous month. If such difference is allocated within an acceptable range, a report will be generated. On the contrary, when the difference is significant, further analyses will be carried in section matrix analysis.

- Matrix analysis. In this section of algorithm matrix containing clearing bids as of the current period (e.g. the current week) will be compared with the ones as of the previous period (e.g. the previous week). Then if the table values are similar (i.e. allocated within an acceptable range), the algorithm will prepare a report, whereas if the differences between the matrixes are significant, the values of particular tables will be deducted from each other; further, a detailed analysis will be conducted and completed with preparation of a final report containing alerts, warnings and figures. 


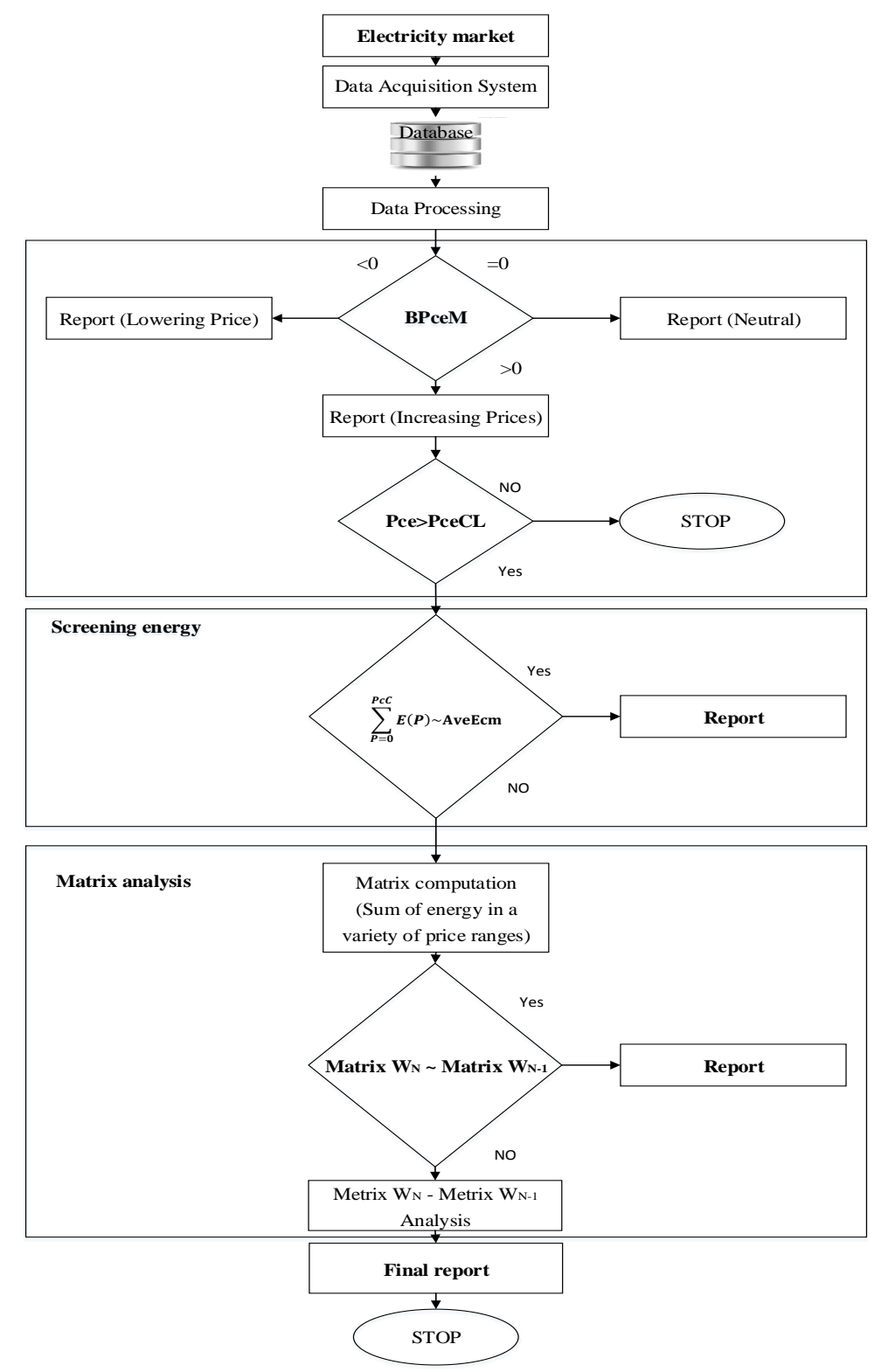

Figure 5. Screening thresholds algorithm

\section{Conclusion}

In this paper, a new structure of electricity market monitoring is developed. This structure has been created for Spanish market, however, it may be applied for monitoring of any electricity market, provided that the relevant source data is available. By the developed system structure, it is possible to compare the parameters examined viavarious methods in order to eliminate any mistakes. Furthermore, the system encompasses a sub-module, which is responsible for the self-diagnosis of measurement and for self-calibration.

The paper also proposes new indexes. These indexes are helpful in fast identification of the technologies or agents responsible for a change of final energy price. One of the crucial elements of proposed algorithms are reference models, parameters defining the competitive price and the threshold values defining market anomalies. The right choice of these parameters will have a significant influence on obtaining the correct results of the analysis. Further, one of 
the more complex elements of the process is to create market simulation models that will require a complex estimation procedures of the market functioning.

As regards the presentation of the structure and analytical methods of monitoring system, the future work will be concerned with the detailed representation of the sub-modules and practical implementation of the system. In order to achieve this, primarily a group of reference models should be construed as well as the optimal parameters of threshold should be determined in detail. The validation of the future system, along with methods and indexes applied to it, will constitute one of the key factors for the correct functioning of the system.

\section{Acknowledgement}

The article is the result of the research project "Determinants of ability to innovation absorption in the tourism industry in relation to renewable energy" financed by the National Science Centre, Poland (decision no. DEC-2012/05/B/HS4/00953).

\section{References}

Anic, I. D., Budak, J., Rajh, E. (2016). New Information Economy in Post-Transition Countries: An Economic Approach to Privacy Concern. Transformations in Business \& Economics, 15(2/38), 165-178.

Balitskiy, S., Bilan, Y., Strielkowski, W. (2014). Energy security and economic growth in the European Union. Journal of Security and Sustainability Issues, 4(2), 125-132. DOI: 10.9770/jssi.2014.4.2(2).

Brożyna, J., Mentel, G., Szetela, B. (2016). Influence of double seasonality on economic forecasts on the example of energy demand. Journal of International Studies, 9(3), 9-20. DOI: $10.14254 / 2071-8330.2016 / 9-3 / 1$

Catalina Crisan-Mitra, C., Dinu, V., Postelnicu, P., Dabija, D. C. (2016). Corporate Practice of Sustainable Development on an Emerging Market. Transformations in Business \& Economics, 15(1/37), 228-243.

Dzikevičius, A., Šaranda, S. (2016). Establishing a set of macroeconomic factors explaining variation over time of performance in business sectors. Business: theory and practice, 17(2), 159-166.

ETSO Report, A Review of the Monitoring of Market Power The Possible Roles of TSOs in Monitoring for Market Power Issues in Congested Transmission Systems.

Galarza, R. J., Mqasqas, I. \& David, J. C. (2006). Elements of Design in Local Market Power Screens. Power Systems Conference and Exposition, 2006. PSCE '06. 2006 IEEE PES, $1710-1717$

Gan, D. \& Bourcier, D. V. (2002). A simple method for locational market power screening. IEEE Power Engineering Society Winter Meeting, 434-439.

Ignotas, A., Stasytyte, V. (2012). Efficiency of energy consumption as a base for sustainable energy sector. Business: theory and practice, 17(2), 128-137.

Kasperowicz, R., Štreimikienè, D. (2016). Economic growth and energy consumption: comparative analysis of V4 and the "old" EU countries. Journal of International Studies, 9(2), 181-194. DOI: 10.14254/2071-8330.2016/9-2/14

Limba, T., Guleviciute, G., Romeika, G., Kiskis, M. (2016). E-Business Qualitative Criteria Application: Analysis of Global Market. Transformations in Business \& Economics, 15(2B/38B), 645-659.

Lisin, E., Strielkowski, W. (2014). Modelling new economic approaches for the wholesale 
energy markets in Russia and the EU. Transformation in Business \& Economics, 13(2B), 566-580.

Lisin, E., Sobolev, A., Strielkowski, W., Garanin, I. (2016). Thermal efficiency of cogeneration units with multi-stage reheating for Russian municipal heating systems. Energies, 9(4), article number 269. DOI: 10.3390/en9040269

Patton, D. B. (2003). Overview of market monitoring tools and techniques. IEEE Power Engineering Society General Meeting, p. 517.

Pinczynski, M. \& Kasperowicz, R. (2016). Overview of electricity market monitoring. Economics and Sociology, 9(4), 153-167.

Sole, T. D. \& Bist, G. (1995). Benchmarking in technical information. IEEE Transactions on Professional Communication, 38(2), 77-82.

Strielkowski, W., Lisin, E., Astachova, E. (2017). Economic sustainability of energy systems and prices in the EU. Entrepreneurship and Sustainability Issues, 4(4), 591-600. DOI: 10.9770/jesi.2017.4.4(14) 
Appendix A

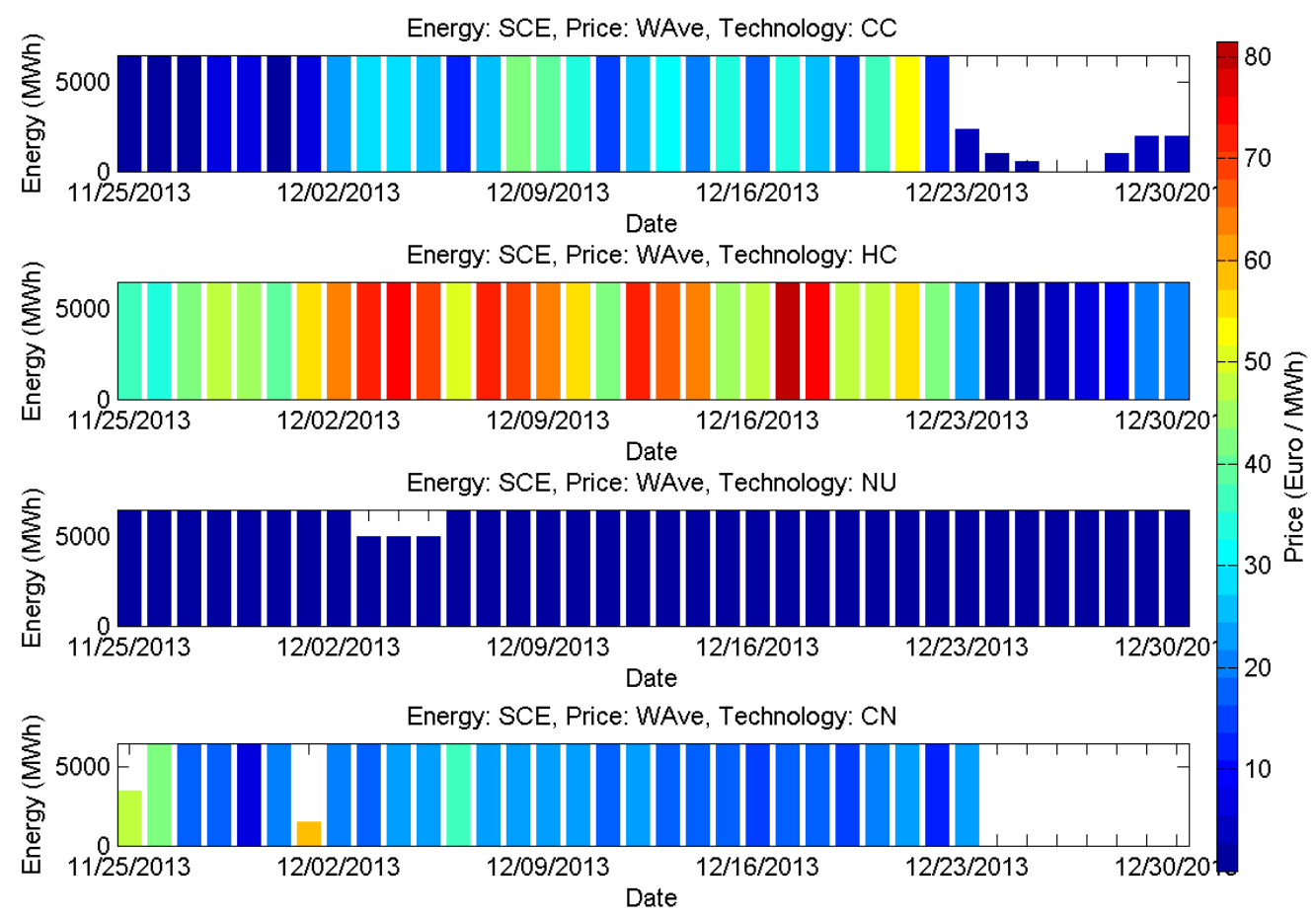

Figure 1. The representation of average weighted energy price divided into technology groups
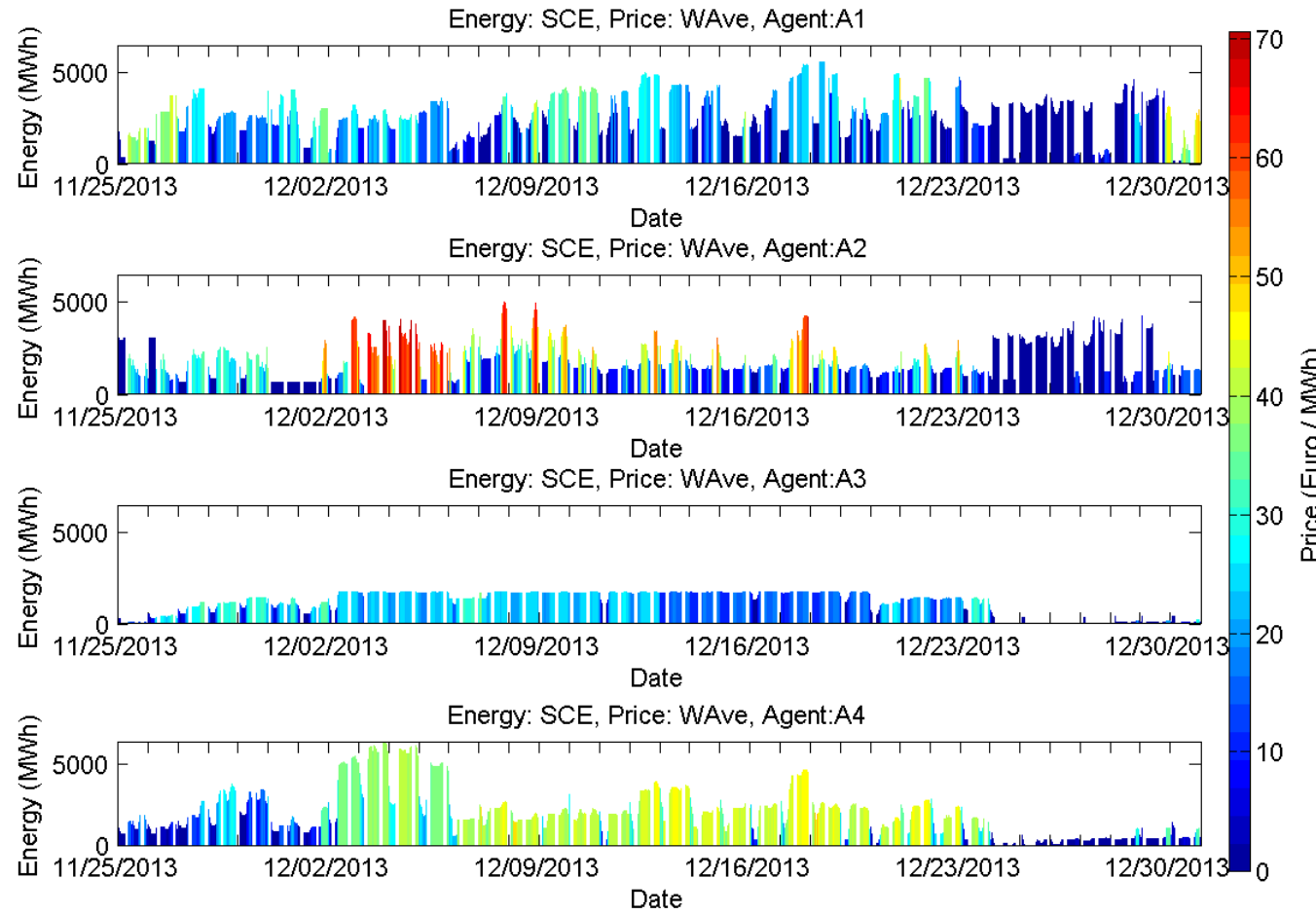

Figure 2. The representation of average weighted energy price divided into agents groups 
Figure 1 presents the following factors: the vertical axis illustrates the amount of cleared energy, the horizontal axis shows the time, the colors represent the average weighted price of energy with regard to the amount of energy, whereas each of the four figures presents different technology (CC-combined cycle technology, HC- hydroelectric, $\mathrm{N}$ - nuclear, $\mathrm{CN}$ - coal). The red color and the color similar to red represent the most expensive energy and could imply that this particular technology contributed to the price increase. Figure 2 is similar to Figure 1 and illustrates four day-ahead agents (A1, A2, A3, A4). It should be noted that the red color could as well indicate the agent whose expensive energy is responsible for the final price increase. 